\title{
Alteraciones hidroelectrolíticas en pacientes con infección por SARS-CoV-2 en el servicio de urgencias de un hospital de segundo nivel de la Ciudad de México
}

\author{
Lilian S. Ramírez-Serrano ${ }^{1}$ Ivan llescas-Martínez ${ }^{\text {* }}$, Zury S. Rosas-Vaquero ${ }^{1}$, Salvador Ávila-Ruiz², \\ Humberto Villanueva-Chávez ${ }^{1}$, Adriana Clemente-Herrera ${ }^{1}$ y Luis F. Paredes-Fernández ${ }^{3}$ \\ ${ }^{1}$ Departamento de Medicina de Urgencias, Hospital General Dr. Enrique Cabrera, Universidad Nacional Autónoma de Mexico; ${ }^{2}$ Facultad de \\ Medicina, Universidad Autónoma de Zacatecas; ${ }^{3}$ Departamento de Cardiología, Hospital General Dr. Enrique Cabrera. Ciudad de Mexico, México
}

\begin{abstract}
Resumen
Introducción: Desde el inicio de la pandemia muchos han buscado dilucidar los mecanismos de esta nueva enfermedad. Una de las áreas que ha tomado gran importancia es el sistema renina-angiotensina-aldosterona y, por tanto, su relación con el equilibrio hidroelectrolítico de los pacientes con enfermedad por coronavirus 2019 (COVID-19). Objetivos: Describir las alteraciones hidroelectroliticas observadas en los pacientes con COVID19 a su llegada al servicio de urgencias. Métodos: Estudio observacional, descriptivo, transversal y retrospectivo. Pacientes con infección confirmada, usuarios del servicio de urgencias. Resultados: De acuerdo con los resultados, el 67.8\% fueron hombres, edad promedio 54.5 años. Se realizo el análisis de electrolitos séricos: concentraciones de sodio $136.1 \mathrm{mEq} / \mathrm{ll}$, potasio $4.315 \mathrm{mEq} / \mathrm{ll}$, cloro $102.3 \mathrm{mEq} / \mathrm{l}$, magnesio $2.222 \mathrm{mg} / \mathrm{dll}$, fósforo $3.702 \mathrm{mg} / \mathrm{dl}$, calcio $8.05 \mathrm{mg} / \mathrm{dl}$, sin diferencias significativas en la población en general. Sin embargo, en el subanálisis de acuerdo con la severidad se encontraron alteraciones en el potasio, cloro, magnesio y fósforo, mientras que en relación con la mortalidad se encontraron diferencias en el potasio, cloro y calcio. Conclusiones: Se encontraron diferencias significativas en las concentraciones séricas de algunos electrolitos que pueden asociarse a cursos de enfermedad más severa o con desenlaces clínicos más desfavorables.
\end{abstract}

Palabras clave: COVID-19. Electrolitos. Sodio. Potasio. Sistema renina-angiotensina-aldosterona.

Hydroelectrolyte alterations in patients with SARS-CoV-2 infection in the emergency department of a second-level hospital in Mexico City

\begin{abstract}
Introduction. Since the beginning of the pandemic, many have sought to elucidate the mechanisms of this new disease. One of the areas that have become very important is the renin-angiotensin aldosterone system and, therefore, its relationship with the water and electrolyte balance of patients with COVID19. Goals. Describe the hydroelectrolyte alterations observed in patients with COVID19 upon arrival at the emergency room. Methods. Observational, descriptive, cross-sectional, retrospective study. Patients with confirmed infection, users of the Emergency service. Results. According to the results, $67.8 \%$ were men, average age 54.5 years. The serum electrolyte analysis was performed, having concentrations of sodium: $136.1 \mathrm{mEq} / \mathrm{L}$,
\end{abstract}

\section{Correspondencia:}

*Ivan Ilescas-Martínez

E-mail: ilescasivan@gmail.com
Disponible en internet: $25-11-2021$

Rev Educ Investig Emer. 2021;3(4):198-206 www.medicinadeemergencias.com

2604-6520 @ 2021 Sociedad Mexicana de Medicina de Emergencias, AC. Publicado por Permanyer México SA de CV. Este es un artículo open access bajo la licencia CC BY-NC-ND (http://creativecommons.org/licenses/by-nc-nd/4.0/). 
potassium: 4,315 mEq/L, chlorine: $102.3 \mathrm{mEq} / \mathrm{L}$, Magnesium: 2,222 mg/dL, Phosphorus: 3,702 mg/dL, Calcium: $8.05 \mathrm{mg} / \mathrm{dL}$, without significant differences in the general population, however, in the sub-analysis according to severity, alterations were found in potassium, chlorine, magnesium, and phosphorus, while concerning mortality differences were found in potassium, chlorine, and match. Conclusions. Significant differences were found in the serum concentrations of some electrolytes that may be associated with more severe disease courses or with more unfavorable clinical outcomes.

Key words: COVID-19. Electrolytes. Sodium. Potassium. Renin angiotensin aldosterone system.

\section{Introducción}

La pandemia iniciada a finales del 2019 secundaria a coronavirus 2 del síndrome respiratorio agudo grave (SARS-CoV-2) es uno de los grandes retos de la salud pública más importantes del último siglo, y a pesar del tiempo que lleva desde sus inicios, esta crisis sanitaria sigue presente en nuestra sociedad cobrando la vida de varios millones de personas en el mundo hoy en día asociadas a esta nueva enfermedad.

Aunque si bien esta nueva enfermedad afecta predominantemente al sistema respiratorio, manifestándose típicamente como neumonía atípica grave que en algunos casos progresa a síndrome de dificultad respiratoria aguda, diversos estudios han mostrado que el espectro de afectación va mucho mas allá de lo pulmonar, siendo también cardiovascular, hepática, neurológica y renal ${ }^{1,2}$. En el ámbito renal algunos autores han propuesto que la enfermedad por coronavirus 2019 (COVID-19) se asocia a otras condiciones como la lesión renal aguda, la cual asociada a COVID-19 presenta cuadros más graves o incremento de la mortalidad'. Otras afecciones relacionadas con el daño renal por COVID-19 son la proteinuria, la hematuria y los desequilibrios hidroelectrolíticos ${ }^{3,4}$.

Lippi, et al. realizaron un metaanálisis para identificar la asociación entre los desequilibrios de los electrolitos y la gravedad de la COVID-19, reportando que la hipopotasemia, la hiponatremia y la hipocalcemia se asociaron a cuadros de COVID-19 grave ${ }^{3}$. Algunos autores proponen diversas causas de las alteraciones electrolíticas en estos pacientes, que van desde la activación del sistema renina-angiotensina-aldosterona alterada por la enzima convertidora de angiotensina 2 regulada negativamente; otra explicación es que la angiotensina 2 activa al receptor tipo 1 de angiotensina y esta induce la reabsorción renal de sodio y agua, llevando así a desequilibrios en la homeostasis del sodio. Otra de las vías que intenta explicar los desequilibrios hidroelectrolíticos es que se ha observado que existe un incremento de las concentraciones de aldosterona que a su vez llevan a un aumento de la excreción urinaria del potasio. $Y$ también se ha observado que el SARS-CoV-2 puede invadir las células de los túbulos renales, causando disfunción tubular, que aporta en la fisiopatología de los desórdenes electrolíticos en los pacientes con COVID-195 ${ }^{5}$. Los primeros reportes de casos mostraban que los trastornos electrolíticos no son tan infrecuentes en los pacientes con COVID-196,7, pero algunos autores han descrito la asociación de los desequilibrios hidroelectrolíticos con la presencia de desenlaces fatídicos o cuadros de COVID-19 grave ${ }^{3,5,8}$. Derivado de esta relación, uno de los componentes importantes de comprender la infección por SARS-CoV-2 es comprender el equilibrio electrolítico que presentan los pacientes al cursar con esta infección, por tanto, el objetivo del presente estudio es describir las alteraciones electrolíticas de los pacientes con enfermedad por SARSCoV-2 en un servicio de urgencias de un hospital de segundo nivel de la Ciudad de México.

\section{Materiales y métodos}

Se trata de un estudio observacional, descriptivo y transversal de tipo retrospectivo. Fue llevado a cabo en un hospital de segundo nivel de la Ciudad en el periodo del 1 de abril al 10 de junio de 2020, previa autorización del comité de ética e investigación de la Secretaría de Salud de la Ciudad de México.

Los criterios de inclusión fueron pacientes con infección confirmada por reacción en cadena de la polimerasa con transcriptasa inversa, usuarios de los servicios de urgencias del Hospital General Dr. Enrique Cabrera y que necesitaron hospitalización, con expediente clínico completo y con reporte de laboratorio de electrolitos séricos completos por el laboratorio clínico del hospital. Los datos clínicos y epidemiológicos se obtuvieron de los registros médicos de los pacientes.

El objetivo principal de este estudio es describir las alteraciones electrolíticas de los pacientes con infección por SARS-CoV-2. Como objetivos específicos se analizaron las diferencias entre los pacientes con enfermedad moderada y grave de acuerdo con el CALL Score $(C=$ comorbidity, $A=$ age, $L=$ lymphocyte 
count, $L=$ lactate dehydrogenase), y las diferencias encontradas entre los pacientes que fueron egresados por mejoría y aquellos que fallecieron durante su estancia hospitalaria.

Se realizo análisis estadístico de tipo descriptivo de las diferentes variables, las variables categóricas se informaron como frecuencias y proporciones, mientras que las variables continuas se describieron utilizando valores de la media, la desviación estándar y el rango intercuartil. Las diferencias entre los grupos estudiados fueron evaluadas mediante análisis de $U$ de Mann-Whitney, debido a que se trata de muestras independientes.

El análisis estadístico se realizo utilizando el paquete estadístico JASP versión 0.14.1.

\section{Resultados}

Se realizo la revisión de 292 expedientes para la obtención de datos, de la revisión de estos expedientes se obtuvieron los siguientes resultados. El $67.8 \%$ de la población analizada fue del sexo masculino (Fig. 1), del total de pacientes un $63.4 \%$ se consideró como un cuadro severo y un $36.6 \%$ como moderado de acuerdo con el índice CALL, en nuestra población no se encontraron pacientes clasificados como cuadros leves por el mismo índice (Fig. 2). La edad promedio en la población general fue de 54.5 años ( \pm 12.99 ), en el subanálisis se encontró que el grupo de pacientes clasificado como severo presentó en promedio una edad más avanzada frente a aquellos con un cuadro moderado (58.22 vs. 48.07 años). Se analizaron algunas variables, gasométricas, citométricas y de química sanguínea, al ingreso se midió la saturación parcial de oxígeno $\left(\mathrm{SpO}_{2}\right)$, cuyo promedio general fue del $75.53 \%$, con una diferencia mínima entre los grupos de pacientes con cuadros moderados y graves (76.01 vs. $75.73 \%$ ). Sin embargo, al realizar el índice $\mathrm{SpO}_{2} /$ fracción inspirada de oxígeno, sí se observaron diferencias discretamente más significativas, con una disminución de 15.8 entre el grupo moderado en comparación al severo (Tabla 1). Gasométricamente se tomo el parámetro de la presión arterial de oxigeno $\left(\mathrm{PaO}_{2}\right)$ en milímetros de mercurio $(\mathrm{mmHg})$, encontrando que el promedio general de la población analizada fue de 54.38; sin embargo, esta se observa una elevación del promedio en el grupo de pacientes severos $(55.88 \mathrm{mmHg}$ ), mientras que los pacientes clasificados como moderados presentaron una cifra menor con $51.78 \mathrm{mmHg}$, con este dato y de acuerdo con la fracción inspirada de oxígeno se calculo el índice de $\mathrm{PaO}_{2} / \mathrm{FiO}_{2}$, encontrando en promedio 245.1, y muestra la misma tendencia a permanecer más elevada en los

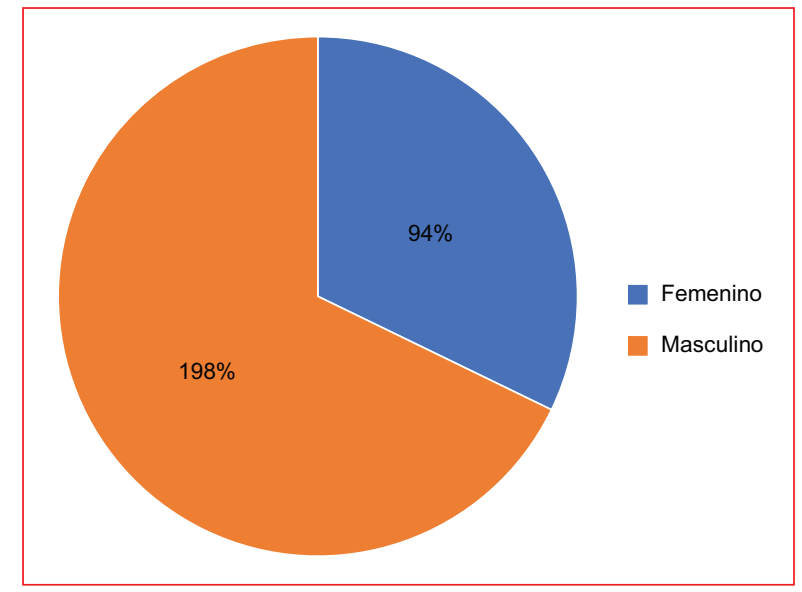

Figura 1. Distribución por sexo de la población analizada.

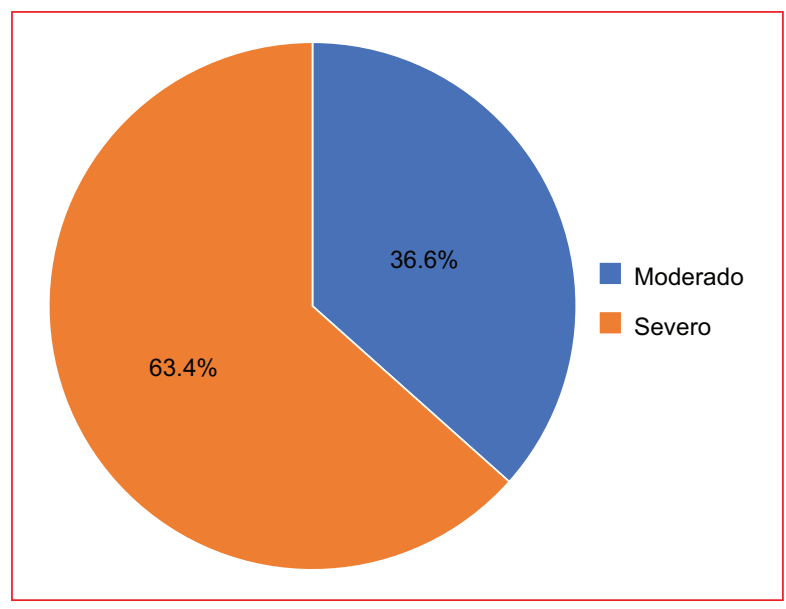

Figura 2. Clasificación de la severidad de los pacientes.

pacientes con clasificación severa que en los moderados (239.9 vs. 248.1). En el análisis gasométrico se encontró que la población en general presentó $2.19 \mathrm{mmol} / \mathrm{l}$, pero en el subanálisis esta cifra se ve más elevada en los pacientes clasificados como severos (2.268 vs. $2.078 \mathrm{mmol} / \mathrm{l})$. En las variables citométricas se encontró: recuento leucocitario $10.6 \times 10^{3}$ en general, siendo ligeramente más elevado en los pacientes severos (10.28 vs. 10.78); en el recuento de neutrófilos, si bien no existió neutrofilia, el predominio neutrofílico fue más marcado en los pacientes con cuadros severos que en aquellos moderados (8.204 vs. 9.484); en cuanto a los linfocitos, la población en general presentó $0.923 \times 10^{3}$, sin embargo, en el subanálisis se encontró que la linfopenia fue más marcada en aquellos pacientes con cuadros severos (0.7082 vs. 1.29); a nivel 
Tabla 1. Caracteristicas Generales de la poblacion estudiada

\begin{tabular}{|c|c|c|c|}
\hline Variable & General & Moderado & Severo \\
\hline $\begin{array}{l}\text { Edad } \\
\text { Promedio } \\
\text { Desviación Estandar }\end{array}$ & $\begin{array}{c}54.5 \\
12.99\end{array}$ & $\begin{array}{l}48.07 \\
10.48\end{array}$ & $\begin{array}{l}58.22 \\
12.87\end{array}$ \\
\hline $\begin{array}{l}\text { Sp02 } \\
\text { Promedio } \\
\text { Desviación Estandar }\end{array}$ & $\begin{array}{l}75.83 \\
12.43\end{array}$ & $\begin{array}{l}76.01 \\
13.85\end{array}$ & $\begin{array}{l}75.73 \\
11.56\end{array}$ \\
\hline $\begin{array}{l}\text { PAFI } \\
\text { Promedio } \\
\text { Desviación Estandar }\end{array}$ & $\begin{array}{l}245.1 \\
110.7\end{array}$ & $\begin{array}{l}239.9 \\
102.6\end{array}$ & $\begin{array}{l}248.1 \\
115.3\end{array}$ \\
\hline $\begin{array}{l}\text { SAFI } \\
\text { Promedio } \\
\text { Desviación Estandar }\end{array}$ & $\begin{array}{l}340.8 \\
80.19\end{array}$ & $\begin{array}{l}350.8 \\
78.76\end{array}$ & $\begin{array}{c}335 \\
80.65\end{array}$ \\
\hline $\begin{array}{l}\text { Pa02 } \\
\text { Promedio } \\
\text { Desviación Estandar }\end{array}$ & $\begin{array}{l}54.38 \\
22.17\end{array}$ & $\begin{array}{l}51.78 \\
20.77\end{array}$ & $\begin{array}{l}55.88 \\
22.85\end{array}$ \\
\hline $\begin{array}{l}\text { Lactato } \\
\text { Promedio } \\
\text { Desviación Estandar }\end{array}$ & $\begin{array}{l}2.19 \\
1.64\end{array}$ & $\begin{array}{l}2.078 \\
1.315\end{array}$ & $\begin{array}{l}2.268 \\
1.806\end{array}$ \\
\hline $\begin{array}{l}\text { Leucocitos } \\
\text { Promedio } \\
\text { Desviación Estandar }\end{array}$ & $\begin{array}{l}10.6 \\
5.45\end{array}$ & $\begin{array}{l}10.28 \\
3.497\end{array}$ & $\begin{array}{l}10.78 \\
6.324\end{array}$ \\
\hline $\begin{array}{l}\text { Neutrofilos } \\
\text { Promedio } \\
\text { Desviación Estandar }\end{array}$ & $\begin{array}{c}9.015 \\
5.26\end{array}$ & $\begin{array}{l}8.204 \\
3.348\end{array}$ & $\begin{array}{l}9.484 \\
6.058\end{array}$ \\
\hline $\begin{array}{l}\text { Linfocitos } \\
\text { Promedio } \\
\text { Desviación Estandar }\end{array}$ & $\begin{array}{c}0.923 \\
0.51\end{array}$ & $\begin{array}{c}1.294 \\
0.4919\end{array}$ & $\begin{array}{l}0.7082 \\
0.3929\end{array}$ \\
\hline $\begin{array}{l}\text { Plaquetas } \\
\text { Promedio } \\
\text { Desviación Estandar }\end{array}$ & $\begin{array}{c}257.4 \\
114\end{array}$ & $\begin{array}{l}269.9 \\
128.2\end{array}$ & $\begin{array}{l}250.1 \\
104.7\end{array}$ \\
\hline $\begin{array}{l}\text { Hemoglobina } \\
\text { Promedio } \\
\text { Desviación Estandar }\end{array}$ & $\begin{array}{c}15 \\
2.08\end{array}$ & $\begin{array}{c}15.66 \\
2\end{array}$ & $\begin{array}{c}14.61 \\
2.04\end{array}$ \\
\hline $\begin{array}{l}\text { Glucosa } \\
\text { Promedio } \\
\text { Desviación Estandar }\end{array}$ & $\begin{array}{l}259.5 \\
187.8\end{array}$ & $\begin{array}{l}232.1 \\
97.61\end{array}$ & $\begin{array}{l}275.4 \\
222.7\end{array}$ \\
\hline $\begin{array}{l}\text { Creatinina } \\
\text { Promedio } \\
\text { Desviación Estandar }\end{array}$ & $\begin{array}{l}1.29 \\
1.15\end{array}$ & $\begin{array}{c}0.929 \\
0.4642\end{array}$ & $\begin{array}{l}1.505 \\
1.363\end{array}$ \\
\hline $\begin{array}{l}\text { Deshidrogenasa lactica } \\
\text { Promedio } \\
\text { Desviación Estandar }\end{array}$ & $\begin{array}{l}547.1 \\
638.7\end{array}$ & $\begin{array}{l}469.8 \\
236.4\end{array}$ & $\begin{array}{l}591.9 \\
779.5\end{array}$ \\
\hline $\begin{array}{l}\text { Proteina C Reactiva } \\
\text { Promedio } \\
\text { Desviación Estandar }\end{array}$ & $\begin{array}{c}22.03 \\
9.53\end{array}$ & $\begin{array}{l}19.63 \\
8.901\end{array}$ & $\begin{array}{l}23.41 \\
9.632\end{array}$ \\
\hline
\end{tabular}

Sp02: saturacion parcial de oxigeno; PAFI: relacion presion arterial de oxigeno/ fracción inspirada de oxigeno; SAFI: relacion saturacion parcial de oxigeno/ fraccion inspirada de oxigeno; $\mathrm{PaO2}$ : presion arterial de oxigeno.

plaquetario y de la concentración de hemoglobina no se observaron alteraciones importantes en la disminución de estas ni en la población general, como en el subanálisis. En el análisis de química sanguínea se encontró que el promedio de concentración de glucosa sérica en la población en general estaba por fuera de las metas de control glucémico del paciente hospitalizado, $259.5 \mathrm{mg} / \mathrm{dl}$, y esta aún es mayor en los pacientes con cuadro severo, en el cual la concentración sérica se eleva a $275.4 \mathrm{mg} / \mathrm{dl}$ en comparación con los cuadros moderados de la enfermedad, que presentaron $232.1 \mathrm{mg} / \mathrm{dl}$. En las pruebas de funcionamiento renal se encontró una elevación de la creatinina sérica mayor en aquellos pacientes con severidad (1.505 vs. 0.929). En cuanto a la deshidrogenasa láctica se encontró una elevación marcada en la población general (547.1), y esta fue más marcada en los pacientes con severidad que en aquellos con cuadro moderado (469.8 vs. 591.9), como parte del análisis de los reactantes de fase aguda se encontró una elevación en general de la proteína $C$ reactiva con un promedio de 22.03, y así mismo como en otros marcadores se encontró una elevación mayor en aquellos con cuadros severos (23.41 vs. 19.63). Las características generales de la población se exponen en la tabla 1.

Al ingreso a urgencias de la unidad hospitalaria se solicitaron electrolitos séricos completos: sodio, potasio, cloro, magnesio, fósforo y calcio. Se realizó el análisis por subgrupos: pacientes con cuadro moderado y severo, pacientes que tuvieron un egreso por mejoría clínica y aquellos que resultaron en alta por defunción hospitalaria.

En el análisis general, se encontró al sodio con un promedio de $136.1 \mathrm{mEq} / \mathrm{l}( \pm 6.952)$, el potasio en promedio general se encontró con una concentración sérica de $4.315 \mathrm{mEq} / \mathrm{l}( \pm 0.97)$, el cloro con un valor promedio general de $102.3 \mathrm{mEq} / \mathrm{l}( \pm 7.125)$, mientras que el magnesio se encontró con un promedio de $2.222 \mathrm{mg} / \mathrm{dl}( \pm 0.5065)$, el fósforo con un valor promedio de $3.702 \mathrm{mg} / \mathrm{dl}( \pm 1.652)$, y para el calcio con un promedio de $8.05 \mathrm{mg} / \mathrm{dl}( \pm 0.5837)$. Todos los valores en el análisis general sin alteraciones sobre los valores de referencia brindados por control de calidad de nuestro laboratorio.

En el subanálisis por grupos de acuerdo con la severidad del cuadro de la enfermedad encontramos al sodio con una disminución entre ambos grupos con $136.639 \mathrm{mEq} / \mathrm{l}$ en los moderados, mientras que los severos presentaron $135.809 \mathrm{mEq} / \mathrm{l}(\mathrm{p}=0.903)$. En el caso del potasio encontramos una elevación de $0.35 \mathrm{mEq} / \mathrm{l}$ entre ambos grupos $(4.089 \mathrm{mEq} / \mathrm{l}$ [moderados] vs. 4.446 [severos]); $p<0.001$ ). En el caso de cloro se encontró una disminución de $3.046 \mathrm{mEq}$ en los 


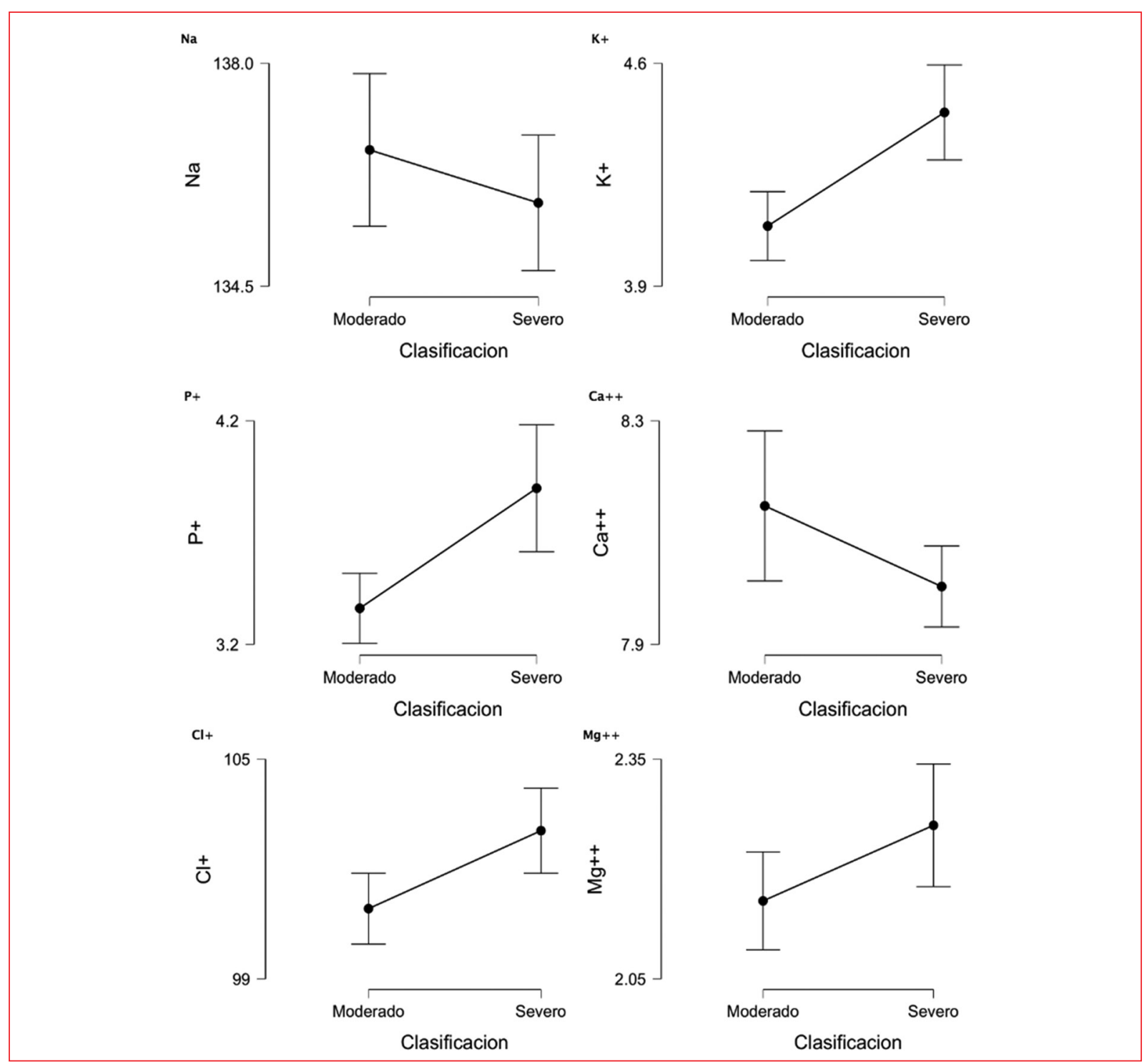

Figura 3. Concentraciones séricas de electrolitos analizadas de acuerdo con la severidad de la enfermedad.

pacientes moderados frente a los severos (100.918 vs. $103.046 \mathrm{mEq} / \mathrm{l} ; \mathrm{p}=0.007$ ). Para los valores observados en las concentraciones séricas de magnesio la diferencia es de $0.104 \mathrm{mg} / \mathrm{dl}$, sin tener una asociación estadísticamente significativa para ambos grupos $(p=$ 0.443), similar a lo observado en las concentraciones séricas de calcio con una diferencia de $0.144 \mathrm{mg} / \mathrm{dl}$ entre el grupo de moderados a severos, sin mostrar una asociación significativa para estos grupos ( $p=0.77$ ) Mientras que en las concentraciones séricas de fósforo sí se mostró una diferencia más significativa entre el grupo de pacientes moderados a severos, con una diferencia de $0.536 \mathrm{mg} / \mathrm{dl}$ (3.362 vs. $3.898 \mathrm{mg} / \mathrm{dl} ; \mathrm{p}=$ 0.003) (Tabla 2) (Fig. 3).
En el subanálisis de acuerdo con el tipo de desenlace clínico se encontraron diferencias más significativas en las concentraciones séricas de electrolitos. En el sodio se encontró una diferencia de 1.552 mayor en aquellos que fallecieron que en los recuperados (135.459 vs. 137.011; $p=0.002$ ); en el valor de las concentraciones de potasio se encontró una diferencia de $+0.297 \mathrm{mEq} / \mathrm{l}$ entre los fallecidos vs. los recuperados (4.19 vs. 4.487; $p=0.001)$. Las concentraciones séricas de cloro se vieron incrementadas en aquellos que fallecieron con una elevación en promedio de $2.272 \mathrm{mEq} / \mathrm{l}$ (101.317 vs. 103.589; $p=0.001$ ), mientras que en las concentraciones de magnesio se encontró una elevación de 0.161 en aquellos que fallecieron ( 2.154 vs. $2.315 ; p=0.002$ ); 


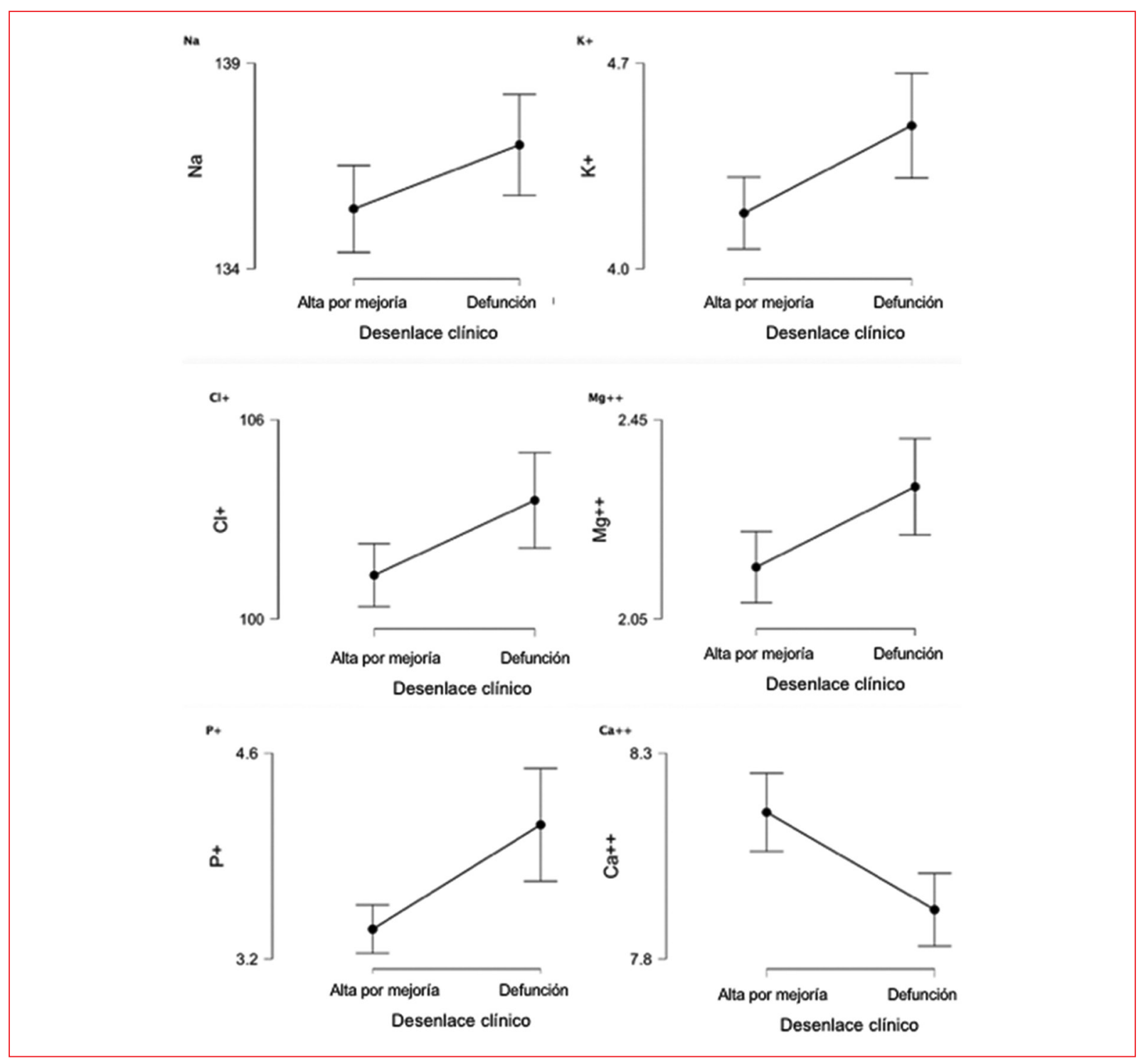

Figura 4. Concentraciones séricas de electrolitos analizadas de acuerdo con el desenlace clínico.

situación similar a la observada en las concentraciones del fósforo, la cual en los fallecidos presenta una elevación de $0.709 \mathrm{mg} / \mathrm{dl}$ (3.403 vs. 4.112; $p=0.055)$, mientras que en las concentraciones plasmáticas de calcio se observa un decremento en aquellos pacientes que fallecieron $(7.92 \mathrm{mg} / \mathrm{dl})$ en relación con aquellos que sobrevivieron (8.156 mg/dl) ( $p<0.001)$ (Tabla 3) (Fig. 4).

$Y$ aunque si bien no se observan desequilibrios hidroelectrolíticos marcados al ingreso, algunos de estos valores nos permiten identificar algunas asociaciones de acuerdo con la severidad del cuadro o incluso que impacten sobre la mortalidad tras el ingreso, sin embargo, el análisis hidroelectrolítico es una situación que se debe de mantener en vigilancia estrecha durante toda la estancia hospitalaria del paciente, ya que la añadidura de fallas orgánicas al cuadro clínico del paciente se pueden traducir en nuevas alteraciones a nivel hidroelectrolítico, lo cual se traduce en incremento de la severidad del cuadro y, per se, de la mortalidad del paciente tras el ingreso hospitalario.

\section{Discusión}

En los estudios iniciales acerca de la COVID-19 se presentaba evidencia que proponía que los pacientes con esta enfermedad padecían algunos desórdenes electrolíticos con respecto a la regulación del sodio, potasio, cloro y calcio ${ }^{3}$. Así mismo, varios estudios de 
Tabla 2. Concentraciones sericas de electrolitos analizadas de acuerdo a la severidad de la enfermedad

\begin{tabular}{|c|c|c|c|c|}
\hline & General & Moderado & Severo & $\mathbf{p}$ \\
\hline $\begin{array}{l}\text { Sodio } \\
\text { Promedio } \\
\text { Desviación } \\
\text { Estandar }\end{array}$ & $\begin{array}{l}136.1 \\
6.952\end{array}$ & $\begin{array}{c}136.639 \\
6.246\end{array}$ & $\begin{array}{c}135.809 \\
7.328\end{array}$ & 0.903 \\
\hline $\begin{array}{l}\text { Potasio } \\
\text { Promedio } \\
\text { Desviación } \\
\text { Estandar }\end{array}$ & $\begin{array}{c}4.315 \\
0.9017\end{array}$ & $\begin{array}{l}4.089 \\
0.563\end{array}$ & $\begin{array}{l}4.446 \\
1.028\end{array}$ & $<0.001$ \\
\hline $\begin{array}{l}\text { Cloro } \\
\text { Promedio } \\
\text { Desviación } \\
\text { Estandar }\end{array}$ & $\begin{array}{l}102.3 \\
7.125\end{array}$ & $\begin{array}{c}100.918 \\
5.05\end{array}$ & $\begin{array}{c}103.046 \\
7.995\end{array}$ & 0.007 \\
\hline $\begin{array}{l}\text { Magnesio } \\
\text { Promedio } \\
\text { Desviación } \\
\text { Estandar }\end{array}$ & $\begin{array}{c}2.222 \\
0.5065\end{array}$ & $\begin{array}{l}2.156 \\
0.348\end{array}$ & $\begin{array}{c}2.26 \\
0.576\end{array}$ & 0.443 \\
\hline $\begin{array}{l}\text { Fosforo } \\
\text { Promedio } \\
\text { Desviación } \\
\text { Estandar }\end{array}$ & $\begin{array}{l}3.702 \\
1.652\end{array}$ & $\begin{array}{l}3.362 \\
0.818\end{array}$ & $\begin{array}{l}3.898 \\
1.956\end{array}$ & 0.770 \\
\hline $\begin{array}{l}\text { Calcio } \\
\text { Promedio } \\
\text { Desviación } \\
\text { Estandar }\end{array}$ & $\begin{array}{c}8.056 \\
0.5837\end{array}$ & $\begin{array}{l}8.148 \\
0.699\end{array}$ & $\begin{array}{c}8.004 \\
0.5\end{array}$ & 0.003 \\
\hline
\end{tabular}

tipo epidemiológico reportaban la coexistencia de esta enfermedad con algunas otras comorbilidades como son la hipertensión, enfermedad renal crónica, diabetes y enfermedades cardiovasculares, entre otras más, aunado a ello en muchas ocasiones los pacientes se complican con la presencia de lesión renal aguda, siendo la suma de todos estos factores un territorio fértil para el establecimiento de los desórdenes hidroelectrolíticos en los pacientes con esta nueva enfermedad.

Aunque si bien en nuestra población estudiada no se observan grandes diferencias en las concentraciones séricas entre los grupos analizados, sí se encuentra una asociación entre los grupos de cuadros severos en el equilibrio del potasio y del cloro. Mientras que para el desenlace clínico también se encontró una asociación entre los niveles de potasio, cloro, calcio y magnesio.

Algunos autores han reportado la hipopotasemia como uno de los potenciales desequilibrios hidroelectrolíticos en la infección por SARS-CoV- $2^{9}$, incluso se ha reportado por algunos autores que la prevalencia de esta condición es de aproximadamente un $62 \%$ de los enfermos ${ }^{9}$. El mecanismo fisiopatológico, aunque si bien no esta del todo dilucidado, se relaciona con el incremento de las concentraciones de aldosterona, que lleva consecuentemente a incrementar la perdida de potasio por la orina ${ }^{3,10}$. Algunos autores coincidían en que las pérdidas gastrointestinales también promovían la perdida de $\mathrm{K}+{ }^{11}$, sin embargo, un estudio sugiere que las perdidas gastrointestinales de potasio son menores en estos pacientes, y que si bien un porcentaje de pacientes presenta síntomas gastrointestinales, no existió diferencia significativa entre el potasio sérico en aquellos que presentaron síntomas gastrointestinales y en aquellos que no, y se demostró que fue mayor la perdida significativa de potasio por la orina ${ }^{9}$. También algunos autores han propuesto que la insuficiencia miocárdica observada en pacientes con esta infección se puede prevenir manteniendo un adecuado nivel plasmático de $\mathrm{K}^{11}$. A pesar de que la hipopotasemia se ha considerado por varios autores como un factor de riesgo para la progresión de la enfermedad, hacen falta más estudios en donde se excluya explícitamente otros factores confusores o que alteren la homeostasis del potasio, tal como medicamentos e intervenciones médicas, y métodos de medición.

Otra condición observada por algunos autores es la presencia de hiponatremia, que está causada por el síndrome de secreción inapropiada de hormona antidiurética. Algunas patologías pulmonares pueden presentar esta patología, como el síndrome de dificultad respiratoria aguda (observado frecuentemente en los pacientes con COVID-19), la neumonía y neoplasias pulmonares; así mismo, el incremento de la producción de citocinas proinflamatorias, como la interleucina 6 , induce también la producción de hormona antidiurética (ADH) por estimulación directa (liberación no osmótica) ${ }^{5}$, esta estimulación indirecta de ADH está mediada por la lesión de la membrana basal alveolar, que resulta en una vía que lleva a la vasoconstricción pulmonar hipóxica. Un grupo de investigadores italianos han asociado la hiponatremia con resultados más graves de la enfermedad ${ }^{12}$.

La hipocalcemia también se ha encontrado como uno de los trastornos hidroelectrolíticos asociados a esta enfermedad, la homeostasis del calcio juega un papel crucial en parte de los eventos fisiopatológicos de la enfermedad, ya que este ion participa de manera importante en la fusión de las membranas y la entrada del virus a la célula ${ }^{13-15}$, ya que se ha observado que tanto el SARS-CoV-2 como el coronavirus causante del síndrome respiratorio de Oriente Medio para realizar la fusión con las célula requieren de procesos moleculares mediados por calcio ${ }^{16}$, y estudios in vitro han demostrado que la fusión de estos con la células huésped se disminuye en algunas líneas celulares que presentan 
Tabla 3. Concentraciones sericas de electrolitos analizadas de acuerdo al desenlace clinico

\begin{tabular}{|c|c|c|c|c|}
\hline & General & Alta por mejoria & Defunción & $\mathbf{p}$ \\
\hline $\begin{array}{l}\text { Sodio } \\
\text { Promedio } \\
\text { Desviación Estandar }\end{array}$ & $\begin{array}{l}136.1 \\
6.952\end{array}$ & $\begin{array}{c}135.459 \\
6.955\end{array}$ & $\begin{array}{c}137.011 \\
6.874\end{array}$ & 0.002 \\
\hline $\begin{array}{l}\text { Potasio } \\
\text { Promedio } \\
\text { Desviación Estandar }\end{array}$ & $\begin{array}{c}4.315 \\
0.9017\end{array}$ & $\begin{array}{c}4.19 \\
0.803\end{array}$ & $\begin{array}{l}4.487 \\
0.999\end{array}$ & 0.001 \\
\hline $\begin{array}{l}\text { Cloro } \\
\text { Promedio } \\
\text { Desviación Estandar }\end{array}$ & $\begin{array}{l}102.3 \\
7.125\end{array}$ & $\begin{array}{c}101.317 \\
6.232\end{array}$ & $\begin{array}{c}103.589 \\
8.039\end{array}$ & 0.001 \\
\hline $\begin{array}{l}\text { Magnesio } \\
\text { Promedio } \\
\text { Desviación Estandar }\end{array}$ & $\begin{array}{c}2.222 \\
0.5065\end{array}$ & $\begin{array}{c}2.154 \\
0.47\end{array}$ & $\begin{array}{c}2.315 \\
0.54\end{array}$ & 0.002 \\
\hline $\begin{array}{l}\text { Fosforo } \\
\text { Promedio } \\
\text { Desviación Estandar }\end{array}$ & $\begin{array}{l}3.702 \\
1.652\end{array}$ & $\begin{array}{l}3.403 \\
1.079\end{array}$ & $\begin{array}{l}4.112 \\
2.149\end{array}$ & 0.055 \\
\hline $\begin{array}{l}\text { Calcio } \\
\text { Promedio } \\
\text { Desviación Estandar }\end{array}$ & $\begin{array}{c}8.056 \\
0.5837\end{array}$ & $\begin{array}{l}8.156 \\
0.623\end{array}$ & $\begin{array}{c}7.92 \\
0.495\end{array}$ & $<0.001$ \\
\hline
\end{tabular}

quelación de calcio intracelular ${ }^{15,16}$. Algunos autores han reportado que la presencia de hipocalcemia se relaciona directamente con la gravedad de la enferme$\mathrm{dad}^{5}$, porque concentraciones más bajas de calcio se relacionan con concentraciones de carga viral más alta, asociada con la duración de la replicación viral ${ }^{5,17}$; otra de las relaciones de las bajas concentraciones séricas de calcio es que los niveles de calcio están relacionados con la función pulmonar y la capacidad de los pulmones de montar una defensa adecuada contra agentes patógenos, y por último y como un efecto sistémico de la hipocalcemia es su relación intrínseca con la desnutrición, condición patológica que se ha comentado por algunos autores como factor de riesgo para el desarrollo de cuadros graves de esta enfermedad.

Por último, uno de los iones ampliamente estudiados por sus funciones inmunitarias es el magnesio, y aunque si bien en nuestros pacientes las alteraciones son mínimas, se conoce bien que el magnesio está implicado en muchos procesos de la función inmunitaria, como la adhesión, la citólisis dependiente de anticuerpos, la unión de linfocitos a inmunoglobulina $\mathrm{M}$, la síntesis de inmunoglobulinas, la respuesta macrofágica y la capacitación y reclutamiento de las células T cooperadoras ${ }^{8,11}$.

\section{Conclusiones}

En la población estudiada se encontró que las diferencias en las concentraciones séricas de potasio, cloro y fósforo mostraron una asociación estadísticamente significativa entre los cuadros severos de los moderados, mientras que en relación con la mortalidad se observaron diferencias entre las concentraciones séricas de potasio, cloro, magnesio y fósforo.

$Y$ aunque si bien no son notorias las alteraciones hidroelectrolíticas al ingreso de los pacientes a los servicios de urgencias, la presencia de los trastornos hidroelectrolíticos impacta de manera notoria sobre el desenlace de algunos pacientes, tal como incrementar los días de estancia hospitalaria, los costos de hospitalización, los costos y recursos de atención, así como el desenlace clínico que pueden cursar estos pacientes.

El estudio de cada uno de los trastornos hidroelectrolíticos con su relación tanto con el evento fisiopatológico como con el desenlace clínico del paciente es un área que requiere de un estudio a mayor profundidad para lograr elucidar los complejos mecanismos que se ven afectados con esta novísima infección.

\section{Financiamiento}

La presente investigación no ha recibido ayudas especificas provenientes de agencias del sector publico, sector comercial o entidades sin ánimo de lucro.

\section{Conflicto de intereses}

Todos los autores declaran no tener ningún conflicto de intereses sobre la presente investigación. 


\section{Responsabilidades éticas}

Protección de personas y animales. Los autores declaran que para esta investigación no se han realizado experimentos en seres humanos ni en animales.

Confidencialidad de los datos. Los autores declaran que han seguido los protocolos de su centro de trabajo sobre la publicación de datos de pacientes.

Derecho a la privacidad y consentimiento informado. Los autores han obtenido el consentimiento informado de los pacientes y/o sujetos referidos en el artículo.

\section{Bibliografía}

1. Wang X, Fang X, Cai Z, Wu X, Gao X, Min J, et al. Comorbid chronic diseases and acute organ injuries are strongly correlated with disease severity and mortality among COVID-19 patients: A systemic review and meta-analysis. Research (Wash D C). 2020;2020:2402961.

2. Kunutsor SK, Laukkanen JA. Renal complications in COVID-19: a systematic review and meta-analysis. Ann Med. 2020;52(7):1-9.

3. Lippi G, South AM, Henry BM. Electrolyte imbalances in patients with severe coronavirus disease 2019 (COVID-19). Ann Clin Biochem. 2020;57(3):262-5.

4. Pei G, Zhang Z, Peng J, Liu L, Zhang C, Yu C, et al. Renal involvement and early prognosis in patients with COVID-19 pneumonia. J Am Soc Nephrol. 2020;31(6):1157-65.

5. Lim JH, Jung HY, Choi JY, Park SH, Kim CD, Kim YL, et al. Hypertension and electrolyte disorders in patients with COVID-19. Electrolyte Blood Press. 2020;18(2):23-30.
6. Guan W, Ni Z, Hu Y, Liang W, Ou C, He J, et al. Clinical characteristics of coronavirus disease 2019 in China. N Engl J Med. 2020; 382(18):1708-20

7. Huang $\mathrm{C}$, Wang $\mathrm{Y}$, Li X, Ren L, Zhao J, Hu Y, et al. Clinical features of patients infected with 2019 novel coronavirus in Wuhan, China. Lancet. 2020;395(10223):497-506.

8. Mabillard H, Sayer JA. Electrolyte disturbances in SARS-CoV-2 infection. F1000Research. 2020;9:587.

9. Chen D, Li X, Song Q, Hu C, Su F, Dai J, et al. Hypokalemia and clinical implications in patients with coronavirus disease 2019 (COVID-19). JAMA Netw Open. 2020;3(6):e2011122.

10. Pal R, Bhansali A. COVID-19, diabetes mellitus and ACE2: The conundrum. Diabetes Res Clin Pract. 2020;162:108132.

11. Taheri M, Bahrami A, Habibi $P$, Nouri F. A review on the serum electrolytes and trace elements role in the pathophysiology of COVID-19. Biol Trace Elem Res. 2021;199(7):2475-81.

12. Berni A, Malandrino D, Parenti G, Maggi M, Poggesi L, Peri A. Hyponatremia, IL-6, and SARS-CoV-2 (COVID-19) infection: may all fit together? J Endocrinol Invest. 2020;43(8):1137-9.

13. Millet JK, Whittaker GR. Physiological and molecular triggers for SARS-CoV membrane fusion and entry into host cells. Virology. 2018;517:3-8.

14. Martens S, McMahon HT. Mechanisms of membrane fusion: disparate players and common principles. Nat Rev Mol Cell Biol. 2008;9(7):543-56

15. Straus MR, Tang T, Lai AL, Flegel A, Bidon M, Freed JH, et al. Ca2+ ions promote fusion of Middle East Respiratory Syndrome coronavirus with host cells and increase infectivity. J Virol. 2020 Jun 16; 94(13):e00426-20.

16. Lai AL, Millet JK, Daniel S, Freed JH, Whittaker GR. The SARS-CoV fusion peptide forms an extended bipartite fusion platform that perturbs membrane order in a calcium-dependent manner. $J$ Mol Biol. 2017;429(24):3875-92.

17. Wu Y, Hou B, Liu J, Chen Y, Zhong P. Risk factors associated with longterm hospitalization in patients with COVID-19: A single-centered, retrospective study. Front Med. 2020;7(June):1-10. 